\title{
Dificultades que enfrentan los nuevos estudiantes universitarios en Matemática
}

\section{Difficulties facing new university students in Mathematics}

\author{
Fabián Eugenio Bravo Guerrero
}

Universidad de Cuenca, Ecuador

Autor por correspondencia: fabianbravo@yahoo.com

Fecha de recepción: 26 de abril del 2019 - Fecha de aceptación: 30 de septiembre del 2019

\section{Resumen}

Esta investigación analiza los problemas que enfrentan los estudiantes durante la transición entre el colegio y el primer semestre de estudios universitarios, en la matemática. Para esto, al final del primer semestre se encuestó a estudiantes recién ingresados, se averiguó sobre su formación previa en matemática, el proceso de ingreso a la universidad y las asignaturas tomadas del área de matemática, además, se revisó los registros académicos una vez finalizado el primer semestre. Se encontró que muchos estudiantes provienen de familias donde los responsables del hogar tienen formación primaria o ninguna; también, que no tienen buenas bases en matemática, y que los estudios en la carrera son difíciles. Se aporta con información para reflexionar sobre el proceso de selección, curso preuniversitario y el diseño curricular de la carrera. Es muy importante conocer a los estudiantes que recién ingresan y su vivencia durante el primer semestre, no solo a través cifras estadísticas, sino comprender las dificultades que ellos atraviesan.

Palabras claves: ingreso a la universidad; formación docente en matemáticas; bases de matemáticas; proceso de admisión; primer semestre en la universidad

\begin{abstract}
This research analyzes the problems that students face during the transition between school and the first semester of university studies, in mathematics. For this, at the end of the first semester, new students were surveyed, it was inquired about their previous formation in mathematics, the process of entrance to the university and the subjects taken in the area of mathematics, in addition the academic records were reviewed once finalized the first semester. It was found that many students come from families where those responsible for the family have no or no primary education; also, that they don't have good bases in mathematics, and that studies in the career are difficult. It is provided with information to reflect on the selection process, pre-university course and the curricular design of the career. It is very important to know the students who have just entered and their experience during the first semester, not only through statistical figures, but to understand the difficulties they go through.
\end{abstract}

Key words: entrance to the university; teacher training in mathematics; bases of mathematics; admission process; first semester in the university 


\section{Introducción}

Esta investigación se refiere al análisis del primer semestre de los estudiantes de la Carrera de Matemáticas y Física de la Universidad de Cuenca. Es un período importante para el estudiante porque atraviesa un proceso de adaptación a la nueva institución educativa, que con frecuencia le puede acarrear dificultades.

La investigación se realizó dado el interés por conocer las dificultades particulares que tiene el estudiante en esta etapa en la Carrera de Matemáticas y Física, la información que proporciona esta investigación, aportará con datos para tomar decisiones sobre la organización del curso preuniversitario, el proceso de selección de aspirantes, y el diseño curricular de la carrera.

Se investigó a los 75 estudiantes de la carrera que ingresaron en septiembre del 2017 mediante una encuesta cuyo instrumento fue un cuestionario estructurado con preguntas cerradas, allí se averiguó sobre su formación previa en matemáticas, el proceso de ingreso a la universidad y su experiencia con las asignaturas del área de matemáticas; también, se revisaron sus registros académicos una vez finalizado el primer semestre.

En este artículo se presenta la revisión literaria, donde se destacan los problemas que el estudiante tiene en su formación secundaria, las dificultades durante el proceso de ingreso a los estudios superiores, y su integración a la vida universitaria, dando énfasis a las dificultades que se tienen con las asignaturas del área de matemáticas. Los resultados muestran cifras que evidencian las dificultades que tienen los estudiantes, y en la discusión se muestran coincidencias con las dificultades de tipo: individuales, institucionales y estatales, mencionadas por Tinto (1989) y que pueden ser factores de deserción.

\section{Dificultades que Enfrentan los Nuevos Estudiantes Universitarios en Matemáticas}

Formar docentes de matemáticas implica una alta responsabilidad, pues los graduados de esta carrera serán quienes enseñen la asignatura en los colegios. Actualmente muchos jóvenes tienen recelo o aversión a la matemática porque en algún momento durante sus estudios tuvieron alguna mala experiencia con la asignatura o el docente; y que, con el paso del tiempo fueron los llevó a desmotivarse y perder el interés en la materia. Como consecuencia de esto se evidencian algunas carencias y vacíos en su formación que repercuten en su apreciación negativa hacia las matemáticas (Bravo, Illescas, Larriva \& Peña, 2017) y las posteriores dificultades en el ingreso y los estudios universitarios.

La matemática es una asignatura importante y muy aplicable a la vida cotidiana de las personas, de manera que tiene sentido estudiarla con motivación y ahínco. La clave está en cómo el docente desarrolla esa clase, dado que es el mediador entre esos contenidos aplicables y los aprendices. Para Gamboa \& Ballestero (2010) la enseñanza debe centrarse en desarrollar habilidades para explorar, visualizar argumentar y justificar, dónde, antes que memorizar, se puedan descubrir y aplicar esos aprendizajes. 
Algunos profesores desarrollan sus clases de matemática de forma tradicional, definiendo conceptos, dictando teoría y resolviendo algún ejemplo en la pizarra; en ese contexto, el estudiante debe recurrir a memorizar formulas, demostraciones o formas de resolver los problemas (Abrate, Delgado \& Pocholu, 2006), ya que esta es la única forma legal de aprobar los exámenes (Goncalves, 2006). En el colegio, los contenidos son presentados como un producto acabado de la actividad matemática (Gamboa \& Ballestero, 2010), que impiden a los estudiantes el razonamiento y la reflexión, se resuelven ejercicios con literales que no tienen ningún significado ni contexto (Bravo, Trelles \& Barrazueta, 2017), manteniéndose los temas como abstractos e inaplicables, con poco o ningún significado para el estudiante.

Dado que en el colegio los docentes requieren dar cuentas del cumplimiento de las planificaciones presentadas, en la práctica se obligan a cumplir con objetivos, antes que centrarse en el logro de aprendizajes por parte de sus estudiantes, así, los estudiantes solo aprenden y memorizan para rendir sus evaluaciones, y luego esos conocimientos ya no son importantes (Bravo, Trelles et al., 2017), esto pesa cuando el estudiante aspira a ingresar a la universidad. No toda institución educativa brinda apoyo a sus estudiantes para orientarles vocacionalmente, además, muchos de ellos aún no han decidido qué estudiarán en la universidad, Aguilar (2007) indica que hay confusión respecto a la elección de carrera y poca información sobre la vida universitaria.

Los escasos cupos que las universidades ofertan son asignados en base al mérito del aspirante (Bravo, Illescas et al., 2017), se otorgan en función de la nota obtenida en el Examen Nacional de Educación Superior (ENES), evaluación que mide las aptitudes de los aspirantes (Guadagni, 2016); además, los aceptados pasan a un curso preuniversitario donde al final rinden una evaluación que incluye temas de lógica y matemática, y que define quienes ingresan a la carrera. Sin embargo, esos cupos no son suficientes para toda la demanda existente, así que muchos aspirantes al carecer de bases, fracasan (Delgado, Santillán, Japón \& Mora, 2018), y terminan siendo excluidos del sistema (Ortega, López \& Alarcón, 2015); otros, acaban resignándose a ingresar en una carrera que no era la primera opción de su preferencia, y probablemente, en el futuro solo llegarán a ser lo que el proceso de selección les permite (de Sousa, Lopes \& Ferreira, 2013).

En América Latina hay dificultades en la educación superior debido a un contexto de desigualdad y pobreza (Ortega et al., 2015), en el Ecuador se han definido políticas que benefician a estudiantes que vienen de sectores menos favorecidos para que puedan acceder al sistema educativo universitario; ellos vienen con un menor capital cultural y con mayores posibilidades de rezagarse y desertar en algún momento (International Institute for Higher Education in Latin America, 2006; Ortega et al., 2015), que podrían inclusive, requerir de la implementación de estrategias para afrontar las carencias formativas que traen del colegio (Ulloa, Gajardo \& Díaz, 2015).

Iniciar los estudios universitarios requiere del nuevo estudiante un proceso de adaptación a la nueva institución que tiene reglas diferentes a la secundaria, deberá desarrollar nuevas maneras de pensar, de estudiar y aprender, también lograr nuevas relaciones sociales y culturales con profesores y compañeros (Aguilar, 2007), estos nuevos espacios y nuevas exigencias suelen conducir a dificultades en el proceso de adaptación, que puede afectar su rendimiento (de Sousa 
et al., 2013). La permanencia del nuevo estudiante depende de lograr un acoplamiento adecuado (Vries, León, Romero \& Hernández, 2011), y para esto, la institución debe planificar actividades que lo integren, porque es importante lograr sentimientos de bienestar personal, social y académico (de Sousa et al., 2013). Tinto (1989) indica que los períodos críticos para el nuevo estudiante son: en el proceso de admisión, el ingreso a la universidad y el primer semestre de estudios, que son los que esta investigación trata.

Las asignaturas de matemáticas son difíciles y un buen número de estudiantes las reprueban y son causa de demoras sus estudios (Solano, Frutos \& Cárceles, 2004), especialmente porque traen deficiencias desde el colegio. Otras dificultades que tienen los estudiantes para rezagarse o desertar es tener dificultades económicas, algunos necesitan trabajar para conseguir recursos y les queda poco tiempo para estudiar (Guevara \& Belelli, 2013), la maternidad también es otra razón que lleva especialmente a las estudiantes a retirarse de sus estudios (Bravo, Illescas et al., 2017).

Una carrera de educación en matemáticas y física, tiene el doble componente: el de las ciencias sociales y de las ciencias experimentales, con profesores que provienen unos de formación técnica, otros con formación pedagógica, y algunos con ambas competencias. Dadas estas diferencias en su formación, algunos profesores aplican métodos tradicionales, que perjudican la calidad de las clases y pone mucha presión a los estudiantes, los exámenes tienen problemas rebuscados, se toman pocas evaluaciones (Bravo, Illescas et al., 2017), además, en algunos casos, las evaluaciones no miden lo aprendido (Bermeo, 2012).

Para los estudiantes, la matemática y geometría son asignaturas complicadas porque en ellas se desarrollan temas abstractos, teóricos y difíciles de comprender (Barrantes \& Blanco, 2005), sin embargo, algunos docentes asumen la compleja tarea de diseñar estrategias que facilitan el desarrollo de la inteligencia de los estudiantes (Goncalves, 2016), el reto es incentivar a los estudiantes a pensar de forma lógica (Gamboa \& Ballestero, 2010), también tienen en mente que no desean reforzar esas tradicionales prácticas ineficientes, porque saben que hay una cultura de enseñanza y aprendizaje distinta que puede ser aplicada (Barrantes \& Blanco, 2005). Ya que los estudiantes están acostumbrados a memorizar y repetir, estas nuevas clases también les representa dificultades, porque requieren usar otras habilidades, como: leer, analizar, razonar, a la que no están acostumbrados.

Las instituciones educativas buscan lograr mejoras en el rendimiento, disminuir los índices de rezago, reprobación y deserción, para hacer más eficiente el proceso educativo (Gutiérrez, Granados \& Landeros, 2011) por eso es importante que se conozca la realidad de cada carrera, con información obtenida de lo que realmente acontece con los estudiantes (Terigi, 2009). En este contexto, la investigación pretende aportar con información de las circunstancias que atraviesan los estudiantes durante su proceso de transición a la universidad y el primer semestre de estudios en la Carrera de Educación en Matemáticas y Física, de modo que desde la institución se puedan definir políticas y planificar el currículo adecuadamente, para evitar que los estudiantes se rezaguen o deserten en esta compleja etapa de su vida estudiantil. 


\section{Metodología}

Esta investigación es un estudio realizado a 75 estudiantes de la Carrera de Educación en Matemáticas y Física, de la Universidad de Cuenca, que tomaron en el primer semestre las asignaturas de Geometría, Trigonometría y Álgebra, se trata de una investigación descriptiva, donde se presentan los resultados y hallazgos de la información proporcionada por los estudiantes acerca de las matemáticas vistos en el colegio, el proceso de ingreso a la carrera y las tres asignaturas del área de matemáticas tomadas en la carrera.

El estudio se realizó en el período comprendido entre septiembre del 2017 a enero del 2018, fueron 38 estudiantes en la jornada matutina y 37 en la jornada vespertina, para obtener la información se aplicó a todos los estudiantes una encuesta estructurada con 55 preguntas cerradas. También se realizó la revisión documental de los registros de asistencia, notas y aprobación de los cursos de Geometría, Trigonometría y Álgebra, asignaturas técnicas que se toman en el primer semestre. Con esta información se realizó un análisis estadístico de tipo descriptivo a las variables investigadas, también se realizó el análisis correlacional de las variables cuantitativas.

\section{Resultados}

Una vez analizada la información proporcionada por los estudiantes de la Carrera de Educación en Matemáticas y Física, esta se organiza en tres momentos diferentes: Información de sus estudios de matemática en el colegio, información del proceso de admisión a la universidad; y, datos del primer semestre en la universidad. Además, se cuenta con datos procedentes del análisis de los registros académicos del primer semestre de estudios en la universidad.

\section{Información general de los estudiantes}

El 54\% son mujeres y el $46 \%$ son varones. El 51\% están en jornada matutina y el $49 \%$ en la jornada vespertina. Un $76 \%$ de los estudiantes viene de colegios fiscales; mientras, el $24 \%$ viene de instituciones educativas particulares o fisco misionales. La mayoría, un $86 \%$ de los estudiantes vienen de colegios ubicados en zonas urbanas y el 14\% de zonas rurales.

Con respecto al nivel de educación del responsable del hogar del que viene el estudiante, se observa que el 38\% de ellos viene de hogares donde el responsable no tiene estudios, o tiene formación primaria, como lo podemos ver en la figura 1; mientras el resto, un $62 \%$ viene de responsables del hogar con formación secundaria o universitaria. 


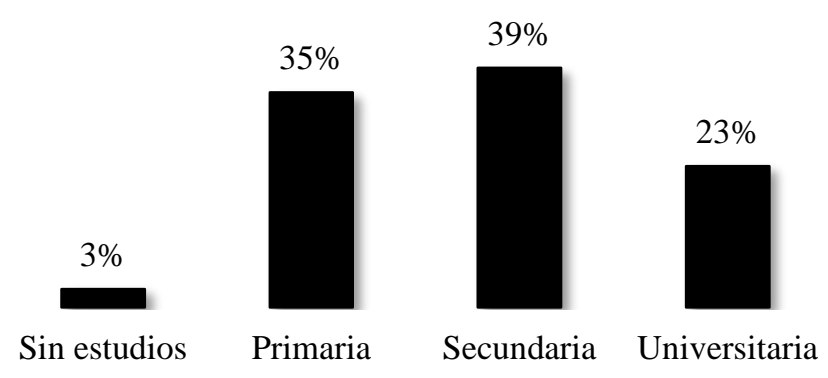

Figura 1. Nivel de educación del responsable de su hogar.

\section{Los estudios de matemáticas en el colegio}

Sobre las clases de matemáticas que recibieron en el colegio, se averiguó sus percepciones y un $92 \%$ de ellos indicaron que les gusta la clase de matemáticas. Un $88 \%$ considera que las clases si eran planificadas por sus profesores.

Los estudiantes indicaron que los recursos más usados por los docentes del colegio en la clase de matemáticas es la pizarra, también usaban instrumentos para el dibujo y se apoyaban en el texto guía, haciendo un limitado uso de otras opciones como materiales didácticos, diapositivas, videos y otros recursos, que pueden apoyar a mejorar la comprensión de la materia, lo podemos ver en la figura 2.

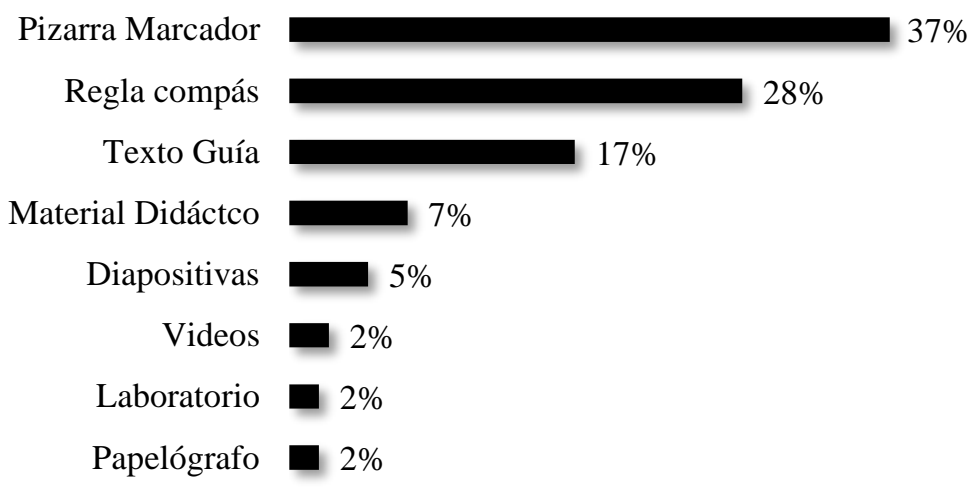

Figura 2. Recursos que el docente usaba en la clase de matemáticas.

Entre las metodologías usadas por los docentes en la clase de Matemáticas destacan prácticas propias del paradigma constructivista, dando importancia a la interacción de los estudiantes con sus compañeros a través del trabajo grupal (31\%), a la participación activa del estudiante en el aula (26\%), en la clase también se realiza investigación (18\%), se desarrollan prácticas en el laboratorio (18\%), experimentos (3\%), salidas de campo (3\%) y otras metodologías (1\%).

Con respecto a las evaluaciones que se toman a los estudiantes en el colegio, se ve en la figura 3 se plantea la resolución de problemas y ejercicios de aplicación modalidades que responden a un aprendizaje contextual, aunque también indican que se les toma definiciones y 
teoría que implica un aprendizaje memorístico y repetitivo. En menor medida se toman ejercicios de tipo teórico, el realizar demostraciones y otras formas.

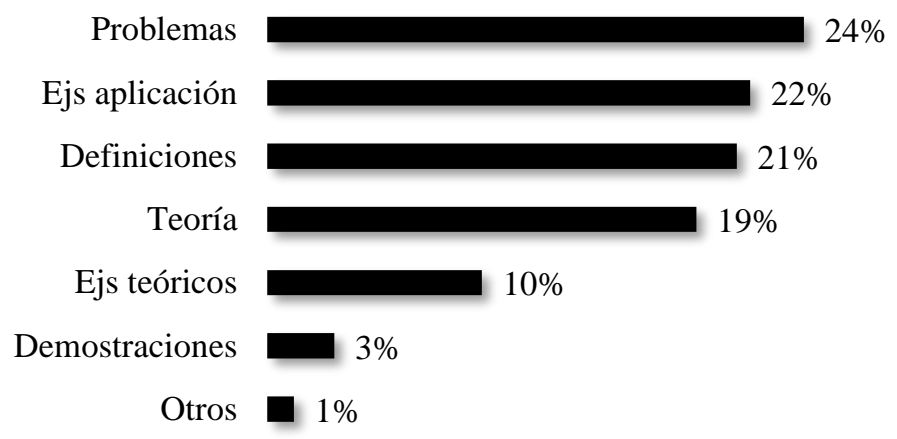

Figura 3. Cómo eran estructuradas las evaluaciones en el colegio.

La nota de aprovechamiento alcanzado por los estudiantes en el colegio para su graduación, fue de 8,59/10 en promedio, con una nota mínima de 7/10, y una máxima de 9,82/10. Según la escala de calificaciones que consta en el Art. 194 del reglamento a la Ley Orgánica de Educación intercultural LOEI (2012), el 71\% alcanza los aprendizajes requeridos porque tiene entre 7 a 8,99, mientras el 29\% domina los aprendizajes requeridos porque tiene entre 9 y 10 , lo podemos ver en la figura 4. En el colegio la asignatura "Matemáticas", engloba los temas de matemática, geometría, trigonometría y estadística.

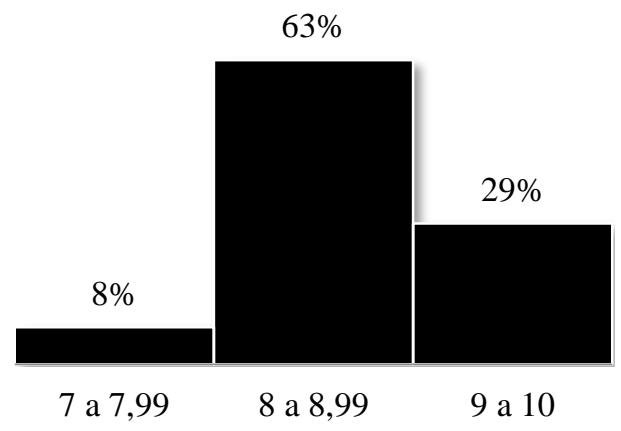

Figura 4. Notas con que los estudiantes se graduaron del colegio sobre 10.

Del análisis correlacional entre el nivel de educación del jefe del hogar con la nota de graduación, se encuentra una moderada relación positiva (Coeficiente de Correlación Lineal de Pearson $r=0,388$ ), es decir, a mayor escolaridad del responsable del hogar la nota de graduación del estudiante es más alta.

\section{En el proceso de admisión a la Universidad}

Una vez graduados, el $80 \%$ de los estudiantes que ingresaron a la Carrera de Matemáticas y Física tiene entre 17 y 18 años, El promedio de la nota ENES para ingreso a la carrera de Matemáticas y Física es de 758,4/1000 puntos, con una nota mínima de 657 y una máxima de 967 puntos, véase la figura 5. 


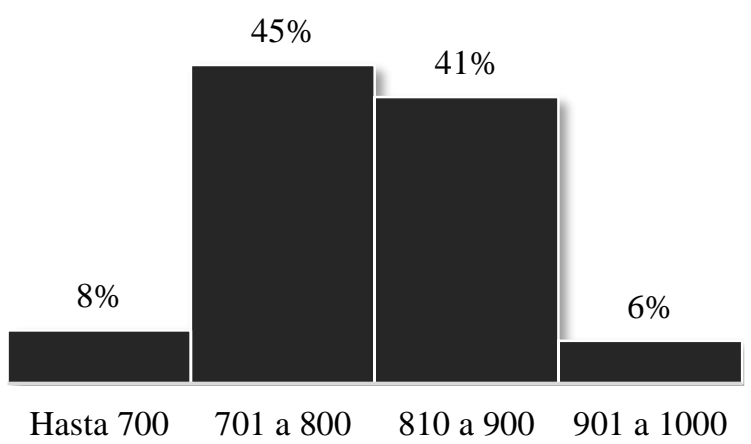

Figura 5. Nota del examen ENES para el ingreso a la Carrera de Matemáticas y Física

Al averiguar sobre la primera opción escogida como preferencia para una carrera, un $43 \%$ de los estudiantes que ingresaron a la Carrera de Matemáticas y Física la tenían como su preferida, un 31\% de ellos querían irse a una carrera técnica (Arquitectura, Ingeniería, Química), y el resto, un $26 \%$, escogieron otras carreras diversas. Al preguntarles por qué ingresaron a la Carrera de Matemáticas y Física, un $60 \%$ de los estudiantes indica que entró por vocación, un $23 \%$ ingresó a la carrera por sugerencia de un familiar o amistad, y el 17\% ingresó a la Carrera de Matemáticas y Física porque no fue aceptado en otra carrera de su preferencia.

Cuando se busca una correlación entre el nivel de educación del jefe del hogar con la nota del Examen Nacional de Educación Superior ENES, se encuentra una moderada relación positiva (Coeficiente de Correlación Lineal de Pearson $r=0,356$ ), esto significa que, mientras más años de escolaridad tiene el responsable del hogar la nota obtenida por el estudiante en el examen ENES es más alta.

\section{El primer semestre en la Universidad}

Una vez ingresados al primer semestre de la Carrera de Matemáticas y Física de la Universidad de Cuenca, un 31\% de los estudiantes indica que la matemática vista en el colegio le sirvió en la carrera. Ningún estudiante considera que la carrera es fácil, sin embargo, en otra pregunta, un $89 \%$ le gusta estudiar en la Carrera de Matemáticas y Física. Podemos ver en la Tabla 1 un resumen del aprovechamiento, donde se evidencia que reprueban en promedio un $33 \%$ de estudiantes en la jornada matutina y un $37 \%$ en la vespertina; con promedios en las tres asignaturas de 62,26/100 en la matutina y 58,03/100 en la vespertina.

Tabla 1

\begin{tabular}{clccc} 
Resultados del primer & \multicolumn{4}{c}{ semestre de los estudiantes de la Carrera de Matemáticas y Física. } \\
\cline { 2 - 5 } Jornada & & $\begin{array}{c}\text { Matemática } \\
\text { Básica }\end{array}$ & Geometría & Trigonometría \\
\hline \multirow{3}{*}{ Matutina } & Matriculados & 39 & 38 & 41 \\
& Reprueban & 17 & 12 & 10 \\
& Promedio /100 & 57,08 & 64,32 & 65,37 \\
\hline \multirow{2}{*}{ Vespertina } & Matriculados & 38 & 37 & 41 \\
& Reprueban & 19 & 14 & 10 \\
& Promedio / 100 & 53,08 & 62,12 & 58,90 \\
\hline
\end{tabular}


Al consultarles acerca de su percepción del primer semestre en la carrera, al $82 \%$ acertó con la elección de la Carrera de Matemáticas y Física, mientras un 18\% de los estudiantes cree que está en la carrera equivocada. De todos los alumnos encuestados, un $78 \%$ considera que ha tenido dificultades en sus estudios. Al preguntarles si se cambiarán de carrera para el siguiente semestre, un $17 \%$ de los estudiantes indica que lo hará.

En la figura 6 podemos ver las dificultades que el estudiante ha tenido durante el semestre: La principal razón es que no tiene buenas bases, esto hace referencia a su deficiente preparación en matemáticas en el colegio; los exámenes son difíciles, es una carrera difícil, no estoy acostumbrado a estudiar tanto, no entiendo a mis profesores, tiene que ver con la dificultad propia de una carrera basada en las matemáticas; tengo dificultades económicas, la distancia a la universidad es un problema, dedico mucho tiempo a las redes sociales, tengo un trabajo, soy padre o madre son dificultades que tienen que ver con factores personales del estudiante.

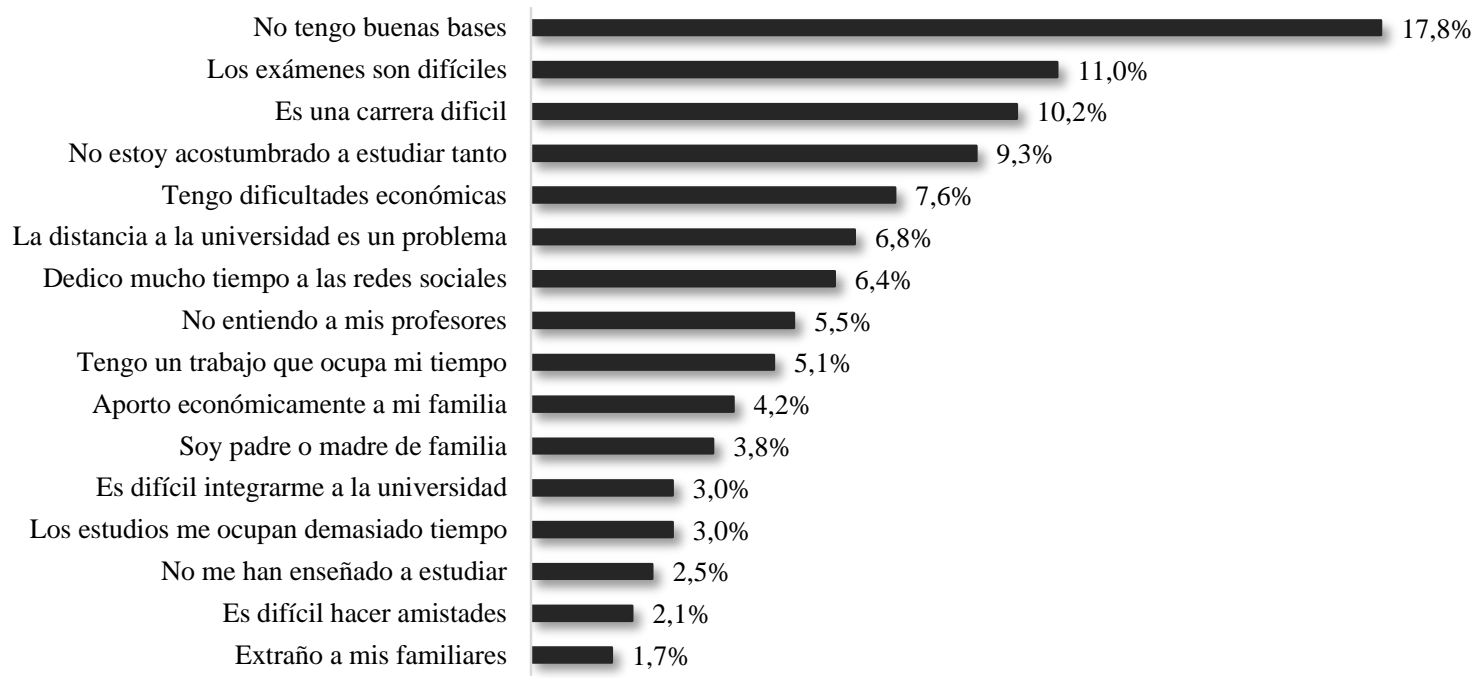

Figura 6. Dificultades que han afrontado los estudiantes durante el primer semestre.

\section{Discusión}

En la prueba para los estudiantes que ingresaron a la Carrera de Matemáticas y Física en septiembre 2015 la nota promedio del examen ENES fue 802 puntos, con 704 mínimo y 933 máximo (Bravo, Illescas \& Quezada, 2018); mientras, en el semestre analizado que inició en septiembre del 2017 el promedio fue 758,4 puntos, con una nota mínima de 657 y una máxima de 967. Este descenso en el promedio se explica porque hasta el año 2016 se exigía que los aspirantes a ingresar a las carreras de educación tengan una nota mínima de 800 puntos, y si el estudiante no había conseguido esa nota, tenía una segunda oportunidad para conseguirla. Para el ingreso de estudiantes a las carreras de educación posterior al 2016 ya no existe una nota mínima, esto repercute en el ingreso de estudiantes con menores calificaciones en el examen ENES; y que, posteriormente pueden tener un menor rendimiento académico como lo señalan Soria-Barreto \& Zúñiga-Jara (2014). 
Respecto al nivel educativo de los padres, García-Castro \& Bartolucci (2007) indican que "a mayor educación de los padres, fue menor el porcentaje de deserción de los hijos" (p.1284), afirmación que se replica en esta investigación, ya que en la carrera de Matemáticas y Física un $62 \%$ de los estudiantes tienen responsables del hogar con formación secundaria y universitaria, por lo que se espera una baja tasa de deserción, consecuente con solo el 17\% de estudiantes que indican que para el próximo semestre se cambiarán de carrera.

El Plan Nacional del Buen Vivir 2013-2017 (2013) indica que se ha incrementado el acceso a la educación superior del $40 \%$ más pobre de la población para romper el círculo de pobreza, y la tasa de asistencia a la educación superior (entre 18 a 24 años), aumentó de 13,54\% en el año 2001, a 22,6\% en el 2010. En el caso de la Carrera de Matemáticas y Física un 38\% de estudiantes provienen de responsables de hogar sin formación o con formación de nivel primario, un dato importante ya que son estudiantes que provienen de familias de estratos sociales menos favorecidos.

En la presente investigación los estudiantes mencionan tener dificultades que coinciden con las categorías mencionadas por Tinto (1989) sobre las causas que llevan a los estudiantes universitarios a desertar de sus estudios: individuales, institucionales y estatales, los porcentajes los podemos encontrar en la figura 6. Las causas individuales son atribuibles al propio estudiante, y pueden ser: los problemas económicos, ser padre o madre de familia, extrañar a la familia, la distancia del hogar a la universidad, dificultad para hacer amistades, tener un trabajo que ocupa el tiempo, aportar económicamente a la familia, e inclusive dedicar mucho tiempo a redes sociales. Las causas institucionales se refieren a la universidad, y pueden ser: la exigencia de la carrera, la dificultad de los exámenes, no comprender a los profesores, que estudiar le ocupe demasiado tiempo, la dificultad para integrarse a la universidad. Las causas estatales se refieren a las políticas educativas para la educación secundaria y pueden ser: el no tener buenas bases en matemáticas, no saber cómo estudiar y no estar acostumbrado a un alto ritmo de estudios. Según Kolko (2013) este suele ser para el estudiante un proceso de reflexión personal y negociación familiar que seguramente tendrá impacto en la vida futura del estudiante.

En una investigación realizada por Bravo, Illescas, Larriva y Peña (2017) en la Universidad de Cuenca, destacan que la maternidad es una dificultad puede llevar a los estudiantes a tener bajos rendimientos y llevarlos a desertar. Del análisis a los registros de notas de Geometría, Matemática básica y Trigonometría en la investigación presente, en un grupo donde hay una madre de familia entre 16 mujeres el promedio es de 62,16/100; mientras, en el otro grupo donde hay 6 madres de familia entre 20 estudiantes mujeres, el promedio es más bajo, de $58,03 / 100$.

\section{Conclusiones}

El estudiante atraviesa una serie de dificultades durante su ingreso a la Carrera de Educación en Matemáticas y Física de la Universidad de Cuenca, para la investigación y análisis de información, se los dividió en tres momentos: las bases que traen del colegio, el proceso de ingreso a la universidad, y su permanencia hasta el final del primer semestre. Respecto a datos de su formación secundaria, el 76\% de ellos viene de colegios fiscales, y solo el 14\% viene de zonas rurales, en la investigación los estudiantes argumentan que las bases que traen desde el colegio 
son deficientes. Los estudiantes que ingresaron a la carrera tuvieron una nota en el examen ENES de 758,4/1000 en promedio. El 38\% proviene de hogares donde el responsable tenía máximo formación primaria, cifra positiva al referirnos a la inclusión de los sectores menos favorecidos a la educación superior. Solo un $43 \%$ de ellos prefirieron la Carrera de Matemáticas y Física, mientras, el $57 \%$ querían estudiar otras carreras, especialmente las técnicas, y no consiguieron un cupo. Sobre su permanencia en la carrera de matemáticas y física el $78 \%$ menciona haber tenido dificultades; en otra pregunta, el 17\% dijo que se cambiará de carrera para el próximo período lectivo. Un 33\% de las asignaturas técnicas fueron reprobadas con promedios que rondan el 60/100, entre las dificultades que enfrentaron en el primer semestre, mencionaron: carencia de bases $(17,8 \%)$, exámenes difíciles $(11 \%)$ y la dificultad de la carrera $(10,2 \%)$, entre las principales razones. Del análisis de correlación, se encuentra una moderada relación entre el nivel educativo del jefe del hogar con las notas obtenidas por el estudiante de graduación del colegio $(\mathrm{r}=0,388)$, así también, con la nota ENES obtenida en el examen para el ingreso a la universidad $(r=0.356)$. La información obtenida aporta con elementos de juicio que permitiría tomar decisiones académicas para minimizar las dificultades para los estudiantes y optimizar los procesos de selección, ingreso, inducción y permanencia especialmente en el primer semestre de los estudiantes de la Carrera de Educación en Matemáticas y Física.

\section{Bibliografía}

Abrate, R., Delgado, G., \& Pochulu, M. (2006). Caracterización de las actividades de Geometría que proponen los textos de Matemática. Revista Iberoamericana de Educación, 39(1), 19.

Aguilar, M. (2007). La transición a la vida universitaria. Éxito, Fracaso, Cambio y Abandono. Proceedings of the fourth Encuentro Nacional de Docentes Universitarios Católicos (ENDUC IV), Universidad y Nación. Camino al bicentenario, Universidad Católica de Santa Fe, Argentina. 18-20.

Barrantes, M., \& Blanco, L. J. (2005). Análisis de las concepciones de los profesores en formación sobre la enseñanza y aprendizaje de la geometría. Números. Revista de Didáctica de las Matemáticas, 62, 33-44.

Barrazueta, J. F., Bravo, F., \& Trelles, C. (2018). Nueva propuesta para realizar una planificación microcurricular en el área de matemáticas. INNOVA Research Journal, 3(9), 76-98.

Bermeo, J. A. (2012). Repitencia y deserción en los primeros años en la Facultad de Ingeniería de la Universidad de Cuenca. (Tesis de Maestría). Universidad de Cuenca. Ecuador.

Bravo, F., Illescas, L. \& Quezada, T. (2018). Proceso de Admisión y Curso de Nivelación en el Ingreso a la Universidad. Un Estudio de Caso. INNOVA Research Journal, 3(10), 134141.

Bravo, F., Trelles, C., \& Barrazueta, J. (2017). Reflexiones sobre la evolución de la clase de matemáticas en el bachillerato ecuatoriano. INNOVA Research Journal, 2(7), 1-12.

Bravo, F., Illescas, L., Larriva, S., \& Peña, M. (2017). Causas de Deserción en el Ingreso a la Universidad; un Estudio de Caso. Revista de la Facultad de Ciencias Químicas, 18, 4859.

Delgado, A., Santillán J., Japón, A., \& Mora, M. (2018). Percepciones de los Aspirantes sobre el Proceso de Admisión a la Universidad Pública Ecuatoriana. INNOVA Research Journal, 3(10), 77-90. 
de Sousa, R., Lopes, A., \& Ferreira, E. (2013). La transición y el proceso de adaptación en la Educación Superior: un estudio con estudiantes de una escuela de enfermería de una escuela de educación. REDU: Revista de Docencia Universitaria, 11(3), 403.

Gamboa, R., \& Ballestero, E. (2010). La enseñanza y aprendizaje de la geometría en secundaria, la perspectiva de los estudiantes. Revista Electrónica Educare, 14(2), 125-142.

García-Castro, G., \& Bartolucci, J. (2007). Aspiraciones educativas y logro académico: un estudio de caso sobre características y condiciones sociales de los estudiantes de la UAM. Revista Mexicana de Investigación Educativa, 12(35), 1267-1288.

Gobierno del Ecuador. (2011). Ley Orgánica de Educación Intercultural. Registro Oficial Ecuador, $2^{\circ}$ suplemento, 127. Quito, Ecuador.

Goncalves, R. (2006). ¿Por qué los Estudiantes no logran un Nivel de Razonamiento en la Geometría? Revista ciencias de la educación, (27), 83-98.

Guadagni, A. A. (2016). Ingreso a la Universidad en Ecuador, Cuba y Argentina. Centro de estudios de la educación argentina, 5(44).

Guevara, H., \& Belelli, S. (2013). Las trayectorias académicas: dimensiones personales de una trayectoria estudiantil. Testimonio de un actor. RevIISE-Revista de Ciencias Sociales y Humanas, 4(4), 45-56.

Gutiérrez, A. G., Granados, D. E., \& Landeros, M. G. (2011). Indicadores de la trayectoria escolar de los alumnos de Psicología de la Universidad Veracruzana. Revista Electrónica" Actualidades Investigativas en Educación", 11(3).

International Institute for Higher Education in Latin America. (2006). Informe sobre la educación superior en América Latina y el Caribe, 2000-2005: la metamorfosis de la educación superior. Iesalc.

Kolko, J. (2013). The Academic Journey a research study about students, education, degree completion, and focus. My Edu Corporation.

Ortega, J. C., López, R. \& Alarcón, E. (2015). Trayectorias escolares en educación superior. Propuesta metodológica y experiencias en México. Veracruz, México.

Secretaría Nacional de Planificación y Desarrollo. (2013). Plan Nacional para el Buen Vivir 2013-2017. Quito, Ecuador.

Solano, J., Frutos, L., \& Cárceles, G. (2004). Hacia una metodología para el análisis de las trayectorias académicas del alumnado universitario. El caso de las carreras de ciclo largo de la Universidad de Murcia. Revista Española de Investigaciones Sociológicas (REIS), 105(1), 217-235.

Soria-Barreto, K., \& Zúñiga-Jara, S. (2014). Aspectos determinantes del éxito académico de estudiantes universitarios. Formación universitaria, 7(5), 41-50.

Terigi, F. (2009). Las trayectorias escolares. Del problema individual al desafío de política educativa. Buenos Aires: Ministerio de Educación.

Tinto, V. (1989). Definir la deserción: una cuestión de perspectiva. Revista de educación superior, 71(71), 33-51.

Trelles, C., Bravo, F., \& Barrazueta, J. F. (2017). ¿Cómo evaluar los aprendizajes en matemáticas? INNOVA Research Journal, 2(6), 35-51.

Ulloa, J., Gajardo, J., \& Díaz, M. (2015). Percepciones sobre la trayectoria socio-académica de estudiantes participantes del Programa Propedéutico de la Universidad de Concepción. Revista de Investigación Educacional Latinoamericana, 52(1), 33-46. 
Vries, W. D., León, P., Romero, J. F., \& Hernández, I. (2011). ¿Desertores o decepcionados? Distintas causas para abandonar los estudios universitarios. Revista de la educación superior, 40(160), 29-49. 